\title{
BUILDING CRACKS: CAUSES AND PREVENTIONS
}

\author{
Anitya S. Zanke \\ Final Year Civil Engineering B.Tech., \\ SSGB College of Engineering and Technology/DBATU, India.
}

\begin{abstract}
Generally, there is no building construction with zero cracks. It's a very common and unavoidable problem in any concrete construction structure. Cracks affect structural safety as well as the beautiful and artistic appearance of any construction. Everyone wishes their construction or building to be crack free, but it is not an easy task or almost impossible. Various types of cracks start to appear on the non-structural and structural part of any construction. These cracks are the result of some unwanted or faulty steps taken during construction. Sometimes, cracks can be a serious concern about the safety of any construction. Cracks do not risk the safety directly, but they encourage some acts which cause major safety problems. Therefore, it is very important to detect cracks before they create any serious safety issue and take preventive measures. However, cracks cannot be completely prevented, but can be controlled by using adequate materials and techniques. The present paper analyses reasons of cracks formation and provides some techniques and measures that can prevent cracks and related issues.
\end{abstract}

Keywords - Concrete, cracks formation, Curing, preventive measures

\section{INTRODUCTION}

A crack in general, means the separation or breaking of anything. A building crack is defined as a full or partial separation of concrete work into two or more parts that are caused due to breaking or fracturing. "Cracks are signs of structural movement, during and after construction. Such movement occurs all the time and usually its magnitude is so small that it passes unnoticed." (Ajagbe W.O. 2018) Cracks bring measurable damage to the safety of any type of construction; it ruins the building architecture, weakens and reduces durability of the structural unity and strength of a building. Cracks that develop due to deterioration of concrete or corrosion or reinforcement bars are the result of unstable land selection, poor construction practices, and inappropriate selection of constituent material and by temperature and shrinkage effects. The owner assumes that something is wrong if he/she sees cracks in some members of the building, especially if the concrete is relatively new. This is not always the case. Some types of cracks are inevitable. Since the cracks cannot be completely removed, one can only try to keep them under control. This is done by properly managing and supervising some major processes such as preparing the subgrade, assuring that the concrete is not too wet, utilizing reinforcement where needed and by properly placing \& spacing crack control joints and expansion joints. However, in some cases cracks can appear in spite of preventive measures taken. If these cracks are active, they show movement in direction, width or depth over a measured period of time. If the cracks are dormant, they remain unchanged. Some cracks do not carry any danger with them, but if remained unrepaired, cracks provide channel for moisture penetration, which can lead to future damage.

\section{TYPES OF CRACKS}

\section{A. Structural Cracks -}

Structural cracks occur due to extra loading, improper construction, and inaccurate design; they can lead to major safety issues of any construction. The cracks in beam, column, slab and foundation are the examples of structural cracks. Structural cracks can be horizontal, vertical, and diagonal. As Arvind Rajabather (2016) rightly classified structural cracks into three types such as cracks in beams (flexural, sheer, torsional, corrosion cracks), cracks in column (horizontal, diagonal, corrosion cracks) and cracks in slab (flexural, shrinkage and corrosion cracks).
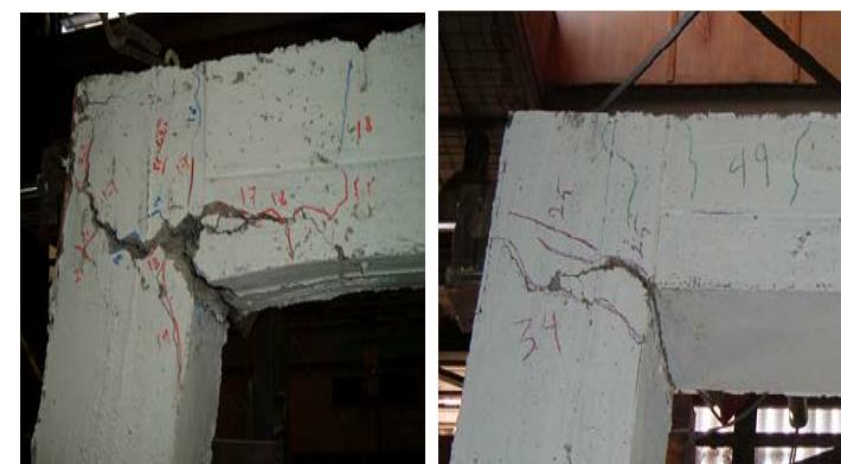

Fig. 1. Structural crack (www.researchgate.net/figure/)

\section{B. Non-Structural Cracks -}

The United States Nuclear Regulatory Commission (2012) has specified various types of non-structural cracks in concrete 


\section{International Journal of Engineering Applied Sciences and Technology, 2020 \\ Vol. 5, Issue 8, ISSN No. 2455-2143, Pages 165-169 \\ Published Online December 2020 in IJEAST (http://www.ijeast.com)}

such as cracks after hardening (Physical, Chemical, thermal, structural which cover drying shrinkage, crazing, corrosion of reinforcement, alkali-aggregate reactions, cement carbonation, freeze, and early thermal contraction) and cracks before hardening (plastic and constructional movement which cover early frost damage, plastic shrinkage and settlement, formwork movement and sub-grade movement). Nonstructural cracks occur because of excessive internal forces developed in the material due to effects of gas, water content, chemical reactions, temperature variation, moisture variation, etc. The cracks in walls, parapet and compound walls, roofs, floor, and driveways are some examples of non-structural cracks. These cracks can be horizontal, vertical, and diagonal.

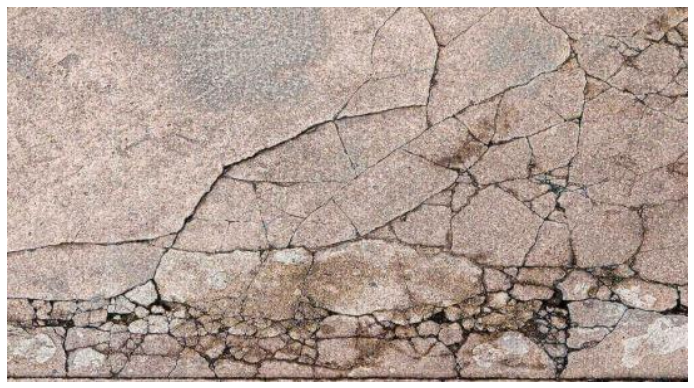

Fig. 2. Non-Structural cracks (gharpedia.com)

Based on the width size of the cracks, Kishor Kunal (2014) has classified cracks into thin, medium and wide. These cracks are explained as follows:

a) Thin cracks:

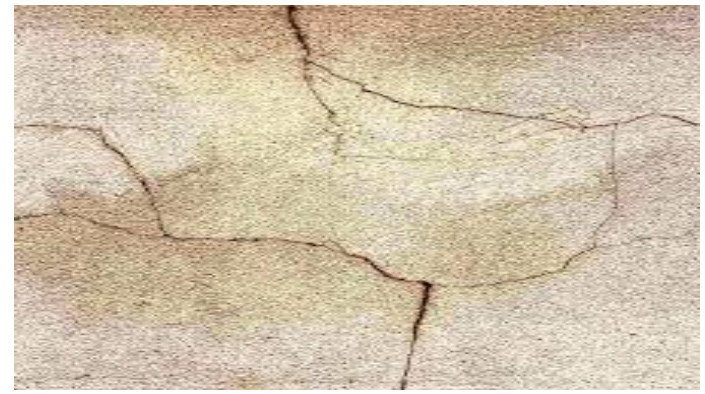

Fig. 3. (a) Thin crack (<1mm) (gharpedia.com)

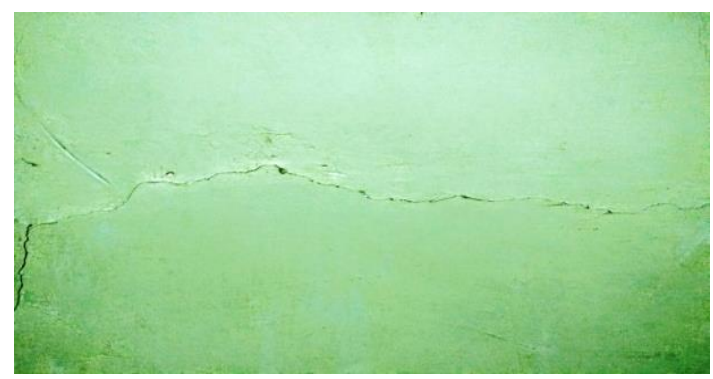

Fig. 3. (b) Thin crack $(<1 \mathrm{~mm})$

The cracks seen in Fig. 3 are thin cracks with less than $1 \mathrm{~mm}$ in width. Theses cracks generally do not cause any structural safety issue as they are the result of some chemical reactions on building materials. These types of cracks are found almost in every construction in spite of preventive measures.

b) Medium cracks:

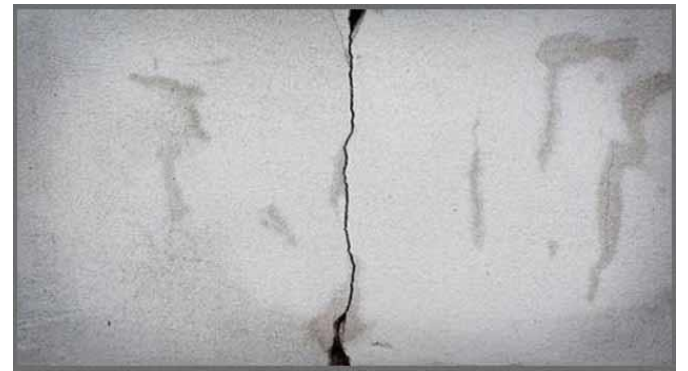

Fig. 4. (a) Medium crack (1-2mm) (constrofacilitator.com)

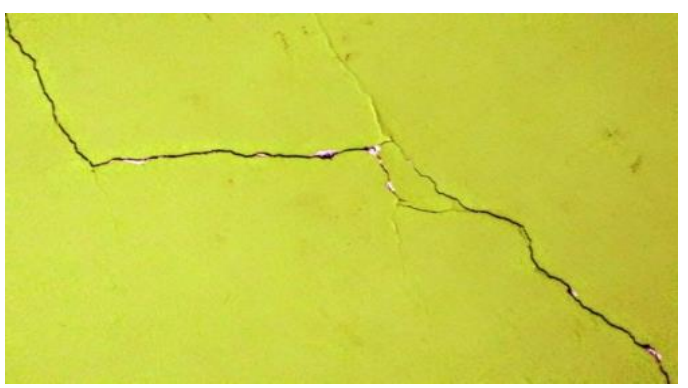

Fig. 4. (b) Medium crack (1-2mm)

The cracks shown in Fig. 4 are of medium size with 1 to $2 \mathrm{~mm}$ in width. These cracks can cause structural safety issues if they appear on structural members and kept unrepaired. They mostly occur due to inaccurate construction design and excess of moisture in building material.

c) Wide cracks:

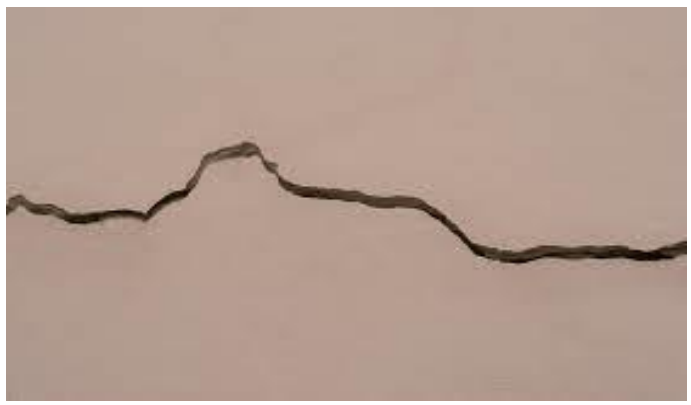

Fig. 5. Wide crack (>2mm) (loveproperty.com)

The crack shown in Fig. 5 is of wide size with more than $2 \mathrm{~mm}$ in width. These cracks appear very clearly visible and need an urgent repair. They mostly occur due to the soil settlement in foundation and elastic deformation of several structural parts of building. 


\section{International Journal of Engineering Applied Sciences and Technology, 2020 \\ Vol. 5, Issue 8, ISSN No. 2455-2143, Pages 165-169 \\ Published Online December 2020 in IJEAST (http://www.ijeast.com)}

\section{CAUSES OF THE CRACKS}

The causes of cracks formation can be discussed as bellow:

a) Elastic Deformation: It occurs due to load in accordance with Hook's law. The elastic deformation in structural components of any building is because of the dead and live loads on that building. "The amount of deformation depends upon elastic modulus, magnitude of loading and dimension of the component." (Government of India, Ministry of Railways, 2004).

b) Thermal Movement: Expansion and contraction of material takes place in accordance with the heating and cooling effect of temperature. It is one of the most potent causes of cracking in buildings; so, it needs much attention. It occurs "regardless of the structure's crosssectional area." (Pooja Nama, 2015)

c) Chemical Reaction: Some chemical reactions may take place because of the "materials used in concrete or when materials come into contact with the concrete after its hardening." (Pooja Nama, 2015). Even though concrete provides a very good protection to the reinforcing steel, there is large number of cases in which corrosion of reinforcement has caused damage to concrete structures just after a year of construction. Increase in volume of materials and internal stresses is also the result of chemical reactions that takes place in building materials that may lead to formation of cracks in building.

d) Creep: Due to the sustained load, a very slow and gradual time dependent deformation occurs in materials that are used in construction work like bricks, cement, mortar, concrete, timbre and marbles. "Creep increases with increase in water and cement content, water cement ratio and temperature. It decreases with increase in humidity of surrounding atmosphere and age of material at the time of loading." (Rishabh Pathak, 2017)

e) Shrinkage: Expansion of any building materials is followed by the absorption of moisture from the surrounding atmosphere, while the shrinkage takes place when the building materials are dry. The shrinkage can be mainly of two types: either it would be a dry shrinkage or a plastic shrinkage. Shrinkage cracks "depend on several factors including temperature, ambient temperature, relative humidity of air, sun and wind, steam velocity inside the concrete mix and water cement ratio." (Kazem Reza Kashyzadeh, 2012)

f) Foundation Movement and Settlement of Soil: Movements in foundation and the soil settlement can be major issue that can lead to formation of hazardous cracks. Formation of shear cracks in the structure generally occur when the foundation undergoes settlement on greater scale, when excessive pressure is loaded on the soil than the safe bearing strength, and when the design of foundation has low factor of safety. If the building is being built on a soil which is not very strong or is not very compact, the settlement of soil starts with excessive water "due to unusual heavy rains or unexpected floods gets into the foundation and causes settlement in the soil under load of the structure. These settlements are not equal in every part of structure, hence result in cracking."'(Chetan Chitte, 2018)

g) Earthquake: Earthquakes occurs when there is some disturbance in the layers of soil or the earth. Cracks can also be the result of earthquakes. The shifting of lower the layers of soil during the earthquake causes cracks in structure. These cracks can turn into very risky cracks if they are more intensive, especially when they are formed in the foundation. Buildings may collapse due to these cracks.

h) Vegetation: The vegetations close to the wall can sometime cause cracks in the wall. The roots of growing trees can penetrate the foundation and walls, and may further develop cracks. "When a tree grows, it simultaneously expands the roots in horizontally in every side and if trees are located close to a wall; these should always be viewed with suspicion. Sometimes plants root begins to grow in the wall itself because of the seeds contained in bird droppings."'(Chetan Chitte, 2018). If timely precaution is not taken on this problem these cracks may grow and cause severe cracking issue.

i) Poor Construction practices: Poor construction practices, may it be mostly because of the carelessness, greed or ignorance, cause unsafe building constructions. Use of poor quality and cheap material, inaccurate mix of mortar and concrete, and improper supervision and poor quality control for monetary gain are some of the major causes of poor building construction.

j) Permeability of concrete: Low permeability is the key to its durability. It is because low permeability helps to keep the aggressive chemicals out of concrete. Therefore, if the permeability of concrete tends to be high then structure will be less durable and will be easily damaged. In order to build a structure with more durability, concrete permeability should be maintained to low. Permeability of concrete can be controlled if factors like water-cement ratio, air voids due to deficient compaction, degree of hydration/curing, etc. are kept under a suitable level.

k) Poor structural designs and specifications: Poor structural design leads to structural risks and cracks formation. Usually engineers of lower scale projects compromise in the structural design to make the construction look more attractive and artistic. Very 


\section{International Journal of Engineering Applied Sciences and Technology, 2020 \\ Vol. 5, Issue 8, ISSN No. 2455-2143, Pages 165-169 \\ Published Online December 2020 in IJEAST (http://www.ijeast.com)}

often, the building loses its durability on the blue print itself and at the time of preparation of specifications for concrete materials and various other related parameters. Therefore, it is also very important that the designer and specifier must first consider the environmental conditions existing around the building site.

1) Extension of existing construction: When new and old construction are joint together while extension of the existing construction, the cracks are bound to come.

Tracing the exact cause of a crack is a very important aspect to ensure the safety of building. "Crack monitoring should be started as soon as possible. The longer the crack monitoring period, the more data will be available for diagnosing the cause." (Roger W. Johnson, 2002)

\section{PREVENTIONS AND REMEDIES}

It is always better to take care and follow safety measures for a crack free construction. "In order to ensure the safety of a structure, it is important to monitor its condition." (Hyunwoo Cho, 2018) There are certain preventions and remedies possible over the problems of cracks. The cracks can always be avoided by using adequate construction material and technique, proper design, and efficient supervision. The following preventive measures should be taken to avoid cracks formation:

1. Use of the maximum size of course aggregate in the mix: this will lower the quantity of water that is used in the mix.

2. The materials used in construction should have minimum moisture movement.

3. Use of composite cement-lime mortar of 1:1:6 mix or weaker for plastering work.

4. Plastering should be done only after the proper curing and fully drying of masonry work.

5. Review of the mix design to ensure that the mix has the lowest water content for workability/performance purposes.

6. The bond between the parapet masonry and concrete slab must be ensured to be very good.

7. Masonry construction on slab should be carried out only after concrete undergoes some drying shrinkage. There should be a time lag of at least 1.5 months between the construction of slab and the masonry work over slab.

8. There should be an adequate time delay between the work of wall masonry and fixing of tiles.

9. Carrying out construction on expansion/contraction joints so that temperature effect can be neutralized.

10. Extent of early drying shrinkage crack can be controlled by the usage of synthetic fibers.

11. Use the chemical admixtures to reduce the amount of dry shrinkage.

12. Very dense concrete or a concrete of a mix of 1:1.5:3 should be used in the concrete foundation if the sulphate content of soil is more than $0.2 \%$ and the sulphate content of ground water is more than $300 \mathrm{ppm}$. A sulphate resisting Portland cement can help if used this issue.

13. Creation of slip joints under the support of concrete slab on walls.

14. Aggregate containing clay, silt and dust must be avoided. "The coarse sand/fine aggregate used in cement concrete and cement mortar mix should has silt and clay less than 4\%." (Shyam Doshi, 2018)

15. Keeping an eye on the vegetation close to the walls and take preventive measure before their root starts penetrating the walls and foundation.

16. If some large trees exist close to a building and these are not causing any problem, as far as possible, do not disturb these trees if soil under the foundation happens to be shrinkable clay.

17. If trees are removed from any construction site and the soil present there is shrinkable clay, then do not carry the activity of construction on that site till the soil gets expanded and stabilized.

18. In an extension work of construction, the old and new should not be bonded, but two separate columns should be constructed.

19. Better to choose a good and well experienced contractor who knows all the problems that can appear in ongoing construction and their solution as well.

20. A detailed and well discussed plan for every part of construction should be made and strictly followed.

\section{TECHNIQUES TO CURE CRACKS}

If someone does not follow the above given instruction, cracks in the building will be the result. Mulla Fayaz (2018) suggested some techniques to be taken in charge to cure the cracks:

- Drilling and plugging: This method is done only when the cracks are in straight line and are accessible at one end. Usually vertical cracks in retaining walls are repaired by this method.

- Epoxy injection: Non-moving cracks can be repaired by this method. Establishing entry and venting ports at close intervals along the cracks, sealing the crack on exposed surfaces, and injecting the epoxy under pressure are the key aspects of this method.

- Routing and sealing: The wideness of cracks is increased in this method with the help of saw and then the groove is filled with a flexible sealant. This technique is used very commonly for treatment of cracks. It can be done on both vertical and curved surfaces.

- Polymer impregnation: Repair of some cracks can be done by using Monomer systems. A liquid consisting of monomers which will polymerize into a solid is called 


\section{International Journal of Engineering Applied Sciences and Technology, 2020 \\ Vol. 5, Issue 8, ISSN No. 2455-2143, Pages 165-169 \\ Published Online December 2020 in IJEAST (http://www.ijeast.com)}

as a monomer system. In this method most commonly used monomer is Methyl methacrylate.

- Stitching: It is used on cracked wall reinforcement and masonry repairs as a permanent repair solution. In this method, holes are being drilled on both sides of cracks, then holes are cleaned and anchoring the legs of the staples in the holes with a non-shrink grout is done.

- Gravity Filling: In this process low viscosity resins and monomers are used to join the cracks whose surface width if from 0.001 to 0.08 inches. Generally, materials like high molecular weight methacrylate, urethanes, and some low viscosity epoxies are used.

- Dry packing: In this method, low water content mortar is placed and then tamping and ramping of the mortar into cracks is done. This results in a very good contact between the existing concrete and the mortar.

\section{CONCLUSION}

To conclude, cracks can cause major structural problems depending on their size and intensity. Also, not all cracks are hazardous to the construction. Some cracks do not cause any structural issue. Though cracks formation cannot be completely prevented, but it can be controlled by using the proper methods and techniques. Before using the prevention techniques, one should know the causes of cracks in detail whether they are due to shrinkage, elastic deformation, earthquakes, and poor construction practices. To control the cracks formation in any construction, some preventions should be taken like preparing a proper work plan of every part of construction considering the environmental factors, using a proper mix of concrete and motor, using maximum size aggregate to lower the water content, creating expansion joints and slip joints where needed, etc. Curing methods such as epoxy ejection, drilling and plugging, gravity filling, stitching, polymer impregnation, routing and sealing, and dry packing should be taken into act if there are large number of cracks in the structure.

\section{REFERENCES}

[1] Ajagbe, W.O., Ojedele, O.S. (2018). "Structural Investigation into the Causes of Cracks in building and Solutions: A Case Study." American Journal of Engineering Research. Vol.7, No. 8, pp.152-160.

[2] Chitte, Chetan and Sonawane Yogesh (2018). "Study on Causes and Prevention of Cracks in Building" IJRASET. Vol. 6, No. III. pp. 453-461.

[3] Cho, Hyunwoo, Hyuk-Jin Yoon, and Ju-yeong Jung. (2018). "Image based Crack Detection using Crack Width Transform (CWT) Algorithm." IEEE Access. Vol. 6. pp. 60100-60114.

[4] constrofacilitator.com/reasons-of-cracks-in-buildings/
[5] Doshi, Shyam et al. (2018). "Methodology for Prevention and Repair of Cracks in Building" Global Research and Development Journal for Engineering. Vol. 3, No. 3. pp. 52-58.

[6] gharpedia.com/blog/classification-of-cracks-nature-widthshape-wise/

[7] GOVERNMENT OF INDIA, MINISTRY OF RAILWAYS (2004). Handbook on Cracks in Building. CAMTECH/2004/C/CRACKS/1.0.

[8] Johnson, Roger W. (2002). "The significance of cracks in low-rise buildings" Structural Survey. Vol.20, No. 5. pp. 155-161. DOI: 10.1108/02630800210456805.

[9] Kashyzadeh, Kazem Reza, and Neda Aghili Kesheh. (2012). "Study type of Cracks in construction and its controlling" International Journal of Emerging Technology and Advanced Engineering. Vol. 2, No.8. pp. 1-4.

[10] Kunal, Kishor and Namesh Killemsetty. (2014). "Study on control of cracks in a Structure through Visual Identification \& Inspection." IOSR Journal of Mechanical and Civil Engineering. Vol. 11, No.5. pp. 64-72. DOI. 10.9790/1684-11566472.

[11] loveproperty.com/news/93257/how-to-fix-cracks-andholes-in-walls/

[12] Mulla, Fayaz. (2018). "Study on Causes \& Control of Cracks in A Structure Building Construction". IJSART. Vol. 4, No. 12. pp. 409-412.

[13] Nama, Pooja, Jain Ankush, Srivastava Rajat and Bhatia Yash (2015). "Study on Causes of Cracks \& its Preventive Measures in Concrete Structures" IJERA. Vol. 5, No. 5. pp. 119-123.

[14] Rajabather, Arvind. (2016). "Investigation of Cracks in Building” Conference: Forensic Structural Engineering. VIT Chennai Campus, Vol. 1. researchgate.net

[15] Rishabh, Pathak and Deepak Rastogi. (2017). "Case Study on Cracks in Public Buildings and their Remedies" International Journal of Science and Research. Vol.6, No. 5. pp. 325-329.

[16] United States Nuclear Regulatory Commission (June 2012). nrc.gov/docs/ML1215/ML12153A317.html 